\title{
Overvoltage by Ferroresonance on a Rural Distribution Feeder: Case Report and Simulation*
}

\section{Sobretensiones por Ferroresonancia en un Sistema de Distribución Eléctrica Rural: Reporte de Caso y Simulación}

\author{
DOI:http://dx.doi.org/10.17981/ingecuc.11.1.2015.03
}

Case Report - Reception Date: June 12, 2014 - Acceptance Date: December 15, 2014

Guillermo F. Di Mauro

Electrical Engineer, Department of Engineering, Universidad Nacional de Mar del Plata (Argentina). gdimauro@fi.mdp.edu.ar

Rubén Ferreyra

Electrical Engineer, Department of Engineering, Universidad Nacional de Mar del Plata (Argentina).roferrey@fi.mdp.edu.ar

Juan A. Suárez

Electrical Engineer, Department of Engineering, Universidad Nacional de Mar del Plata (Argentina). jsuarez@fi.mdp.edu.ar

\author{
Alejandro D. Jurado \\ Electrical Engineer, Department of Engineering, Universidad Nacional de Buenos Aires. \\ Ciudad Autónoma de Buenos Aires (Argentina). ajurado@fi.uba.edu.ar
}

To cite this paper:

G. F. Di Mauro, R. Ferreyra, J. A. Suarez and A. D. Jurado. "Overvoltage by Ferroresonance on a Rural Distribution Feeder: Case Report and Simulation”, INGE CUC, vol. 11, no. 1, pp. 34-47, 2015. DOI: http://dx.doi.org/10.17981/ingecuc.11.1.2015.03

\begin{abstract}
The objective of this work was to analyze an overvoltage case in a rural distribution feeder belonging to an electrical distribution company in the southeast of the Buenos Aires Province in Argentina. The network was modeled in the Electromagnetic Transients Program, based on the electrical parameters that make up the circuit, in order to evaluate its behavior under various switching and load states. The simulation analysis showed that during certain operation and load situations, the conditions for the overvoltage phenomenon occurred, causing a voltage increase in the single-phase transformer feeding. The guidelines for prevention and control of the phenomenon were provided taking into account the results obtained in the study.
\end{abstract}

Keywords - Overvoltage, ferroresonance, rural electrical distribution, fuse opening, ATPDraw, nonlinear circuits.
Resumen-- El objetivo del trabajo presentado fue el de analizar un caso de sobretensión en un sistema de distribución de energía eléctrica rural $(13,2 \mathrm{kV})$ perteneciente a una Cooperativa de Electricidad del sudeste de la Policía de Buenos Aires, Rep. Argentina. A partir de los parámetros que componen el circuito eléctrico se modeló la red dentro del entorno del programa computacional Electromagnetic Transients Program, con el fin de evaluar su comportamiento ante distintas maniobras de interrupción y estados de carga. El análisis de la simulación demostró que, en ciertas situaciones de operación y carga, se conjugaron las condiciones para la ocurrencia del fenómeno de ferrorresonancia, ocasionando la elevación de tensión de alimentación en transformadores monofásicos. Considerando los resultados del estudio, se brindaron pautas a tener en cuenta para la prevención y control del fenómeno.

Palabras claves-- Sobretensiones, Ferroresonancia, Distribución eléctrica rural, Apertura de fusibles, ATPDraw, Circuitos no lineales. 


\section{INTRODUCTION}

The laws governing the distribution of electricity in the region of the Province of Buenos Aires, Argentina (Act 11769/96) imposes quality conditions to the power supply system under the concept of "Electrical product quality" evaluating, among other parameters, the magnitude of the voltage delivered. The distribution service providers are sanctioned by the withdrawal of voltage magnitudes compared to reference values, thus implying unnecessary economic expenditures.

Different circumstances in the operation of a distribution system may cause undesirable voltage values which, although short in time, are likely to become detrimental to both the user and the network equipment itself: lightning, earth fault, equipment operation, etc.

Ferroresonance, as an overvoltage result, occurs in a distribution system by the interaction of capacitance and inductance components present in the circuit. While its appearance is conditioned by the characteristics of the network [1] and simultaneity of factors [2], [3], its consequences can have a high degree of severity [4], [5].

Overvoltage caused by ferroresonance in distribution systems were analyzed from the middle of the last century. Experimental and analytical studies have been conducted to understand and mitigate the phenomenon [1], [6]-[9].

The current evolution of power systems has led to an increase in the occurrence of this phenomenon as a consequence of the use of components that are conducive (shielded cables, single phase switching devices, the use of low load transformers, etc.), which in the field of research arouses a great interest for its study.

In this framework, the paper presents a case report of ferroresonance in a medium voltage rural distribution line $(13,2 \mathrm{kV})$ belonging to an electrical distribution company in the southeast of the Buenos Aires Province in Argentina. In the environment of the Electromagnetic Transients Program (EMTPATPDraw), the involved circuit is modeled and conditions that could have caused the phenomenon are analyzed. The results indicate the feasibility of overvoltage under certain operating conditions, coinciding with reports of technical personnel and contingency accounts of users.

\section{Ferroresonance}

In this section, basic concepts associated with ferroresonance are provided. Specialized literature delves into mathematical and physical details [10], [11].

If we consider a series circuit like Fig. 1 where all parameters are assumed linear, the intensity of the current is given by (1):

$$
\bar{I}=\frac{\bar{U}_{0}}{R+j\left(X_{L}-X_{C}\right)}
$$

where:

$\mathrm{U}_{0}$ : Power Supply /voltage source

$\mathrm{X}_{\mathrm{L}}: 2 \Pi f \mathrm{~L}$ Inductive reactance of circuit

$\mathrm{X}_{\mathrm{C}}: 1 / 2 \pi \mathrm{nC}$ Capacitive reactance of circuit

R: Circuit resistance

If on the same circuit, the magnitude of the electrical capacitance (C) is varied, a plot of the current I can be obtained regarding the capacitive reactance $\left(\mathrm{X}_{\mathrm{C}}\right)$ as shown in Fig. 2.

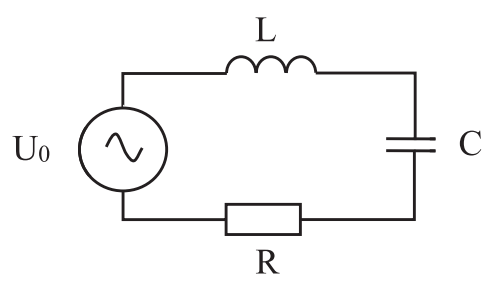

Fig.1. Series circuit RLC. Source: [11]

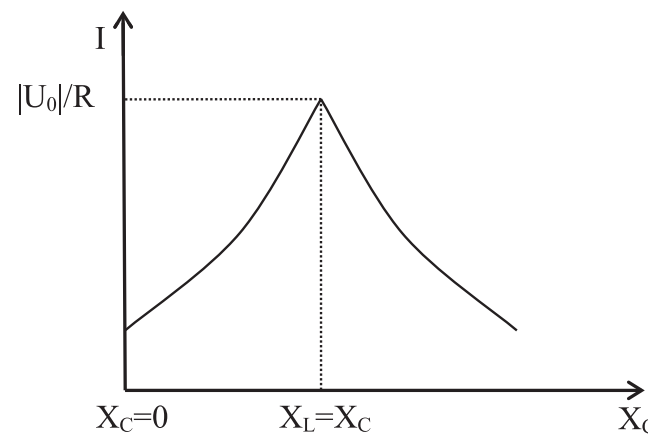

Fig. 2. Resonance in a series circuit RLC with linear inductance. Source: [11]

The maximum current can be expressed by (2).

$$
\operatorname{Imax}=\underset{\mathrm{R}}{\left|U_{o}\right|}(2)
$$

This maximum value is obtained for the series resonance condition $\left(\mathrm{X}_{\mathrm{L}}=\mathrm{X}_{\mathrm{C}}\right)$ and is limited only by the circuit resistance.

This operating state can be the cause of significant increases in voltage in the components of the circuit itself.

In distribution networks, the presence of power transformers gives nonlinear characteristic to the circuit inductance.

If the linear and nonlinear characteristics of the inductances are compared, it is observed that the inductive reactance $\left(\mathrm{X}_{\mathrm{L}}\right)$ associated with the latter acquires an infinite number of values above the saturation elbow of its magnetic core (Fig. 3). 
If the graphic I-Xc is repeated again for the series circuit RLC, but with nonlinear inductance, it can be seen in Fig. 4 there is ample margin of values $\mathrm{Xc}$ for which the resonance condition is satisfied. Indeed, past the saturation elbow, for each value of inductive reactance, there is a capacitive reactance value (given by a respective value of capacity) that matches. Since this situation is strictly due to the presence of ferromagnetic material in the inductance, the phenomenon is called ferroresonance.

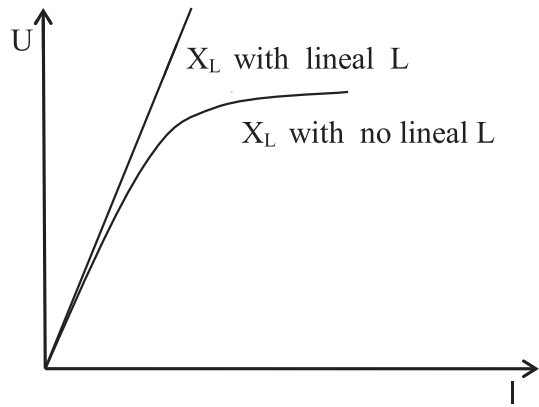

Fig.3. Inductive reactance with linear and nonlinear L. Source: [11]

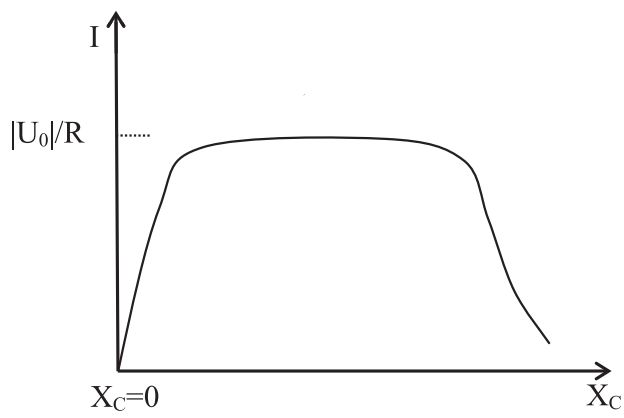

Fig. 4. Resonance in a series circuit RLC with nonlinear inductance. Source: [11]

Fundamental differences of a ferroresonant circuit with respect to a linear resonant circuit are:

- the ability to resonate within a big range of capacitance values.

- the frequency of the voltage and current waves may be different from the excitation.

- the existence of several permanent regimes of operation for a given configuration and parameter values. The achieved regime depends on the initial conditions (electric charges accumulated in the condensers, capacitors or power lines, remaining flux of the material forming the magnetic circuit of the transformer, the connection / disconnection instant, etc. [12].)

The behavior of a ferroresonant circuit can be explained graphically [11].

Assuming the circuit RLC resistance equals to zero (Fig. 5), a steady-state voltage is obtained given by (3) and (4):

$$
\bar{U}_{0}=\bar{U}_{L}+\bar{U}_{C}
$$

This relationship can be written as:

$$
\pm \bar{U}_{L}=\bar{U}_{0}+\frac{\bar{I}}{w C}
$$

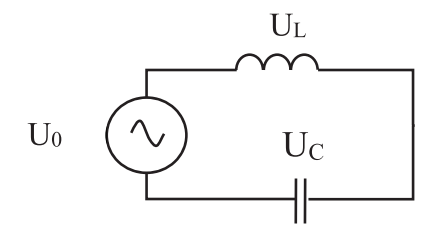

Fig 5. Series Circuit L C. Source: [11]

Where:

$\mathrm{U}_{\mathrm{L}}$ : Voltage drop in the inductance $\mathrm{L}$

$\mathrm{U}_{\mathrm{C}}$ : Voltage drop in the capacitor $\mathrm{C}$

w: $2 \pi \mathrm{f}$

The positive sign (+) corresponds to inductive currents and the negative (-) to capacitive. The three components of this equation are plotted in Fig. 6 .

The right side of (4) is represented by a straight line intersecting the axis of the voltage in the value $\mathrm{U}_{0}$ and its slope is obtained from tan $=1 / \mathrm{wC}$.

The intersection points of the straight lines with the nonlinear characteristic of the inductance $\mathrm{U}_{\mathrm{L}}=\mathrm{f}(\mathrm{i})$ satisfy the relation given by (3.)

For small values of capacitance (line 1), the zone of negative currents (capacitive) only yields a cutoff point. The capacitive reactance is larger than the inductive reactance, resulting in higher than normal voltage in the capacitor.

As the capacitance increases (lower slope of the line), the point of solution is drawn into the saturation elbow of the curve $\mathrm{U}_{\mathrm{L}}=\mathrm{f}(\mathrm{i})$.

For higher capacity values (line 2 ) three intersections can be obtained with curve $\mathrm{U}_{\mathrm{L}}=\mathrm{f}(\mathrm{i})$, with two intersections in the positive current zone (inductive). The intersection marked with $c$ is an unstable operation point that can be reached during a transient. The intersections $a$ and $b$ are stable positions and can exist in steady state.

For points $a$ and $c$, there will be high values of voltage and current. The natural tendency is to reach the position of point $a$ where a small voltage is developed across the capacitor.

To increase the system voltage $\left(\mathrm{U}_{0}\right)$, the capacitor line 2 would move upwards, which in turn removes the operating point $b$ that is reached in the third quadrant as operating zone. Under this condition, the circulating current can reach very high values that cause the voltage to drop and again an operating point is reached as $b$. Phenomena like these are seen in cases of ferroresonance, voltage and current appear to vary randomly [5]. 


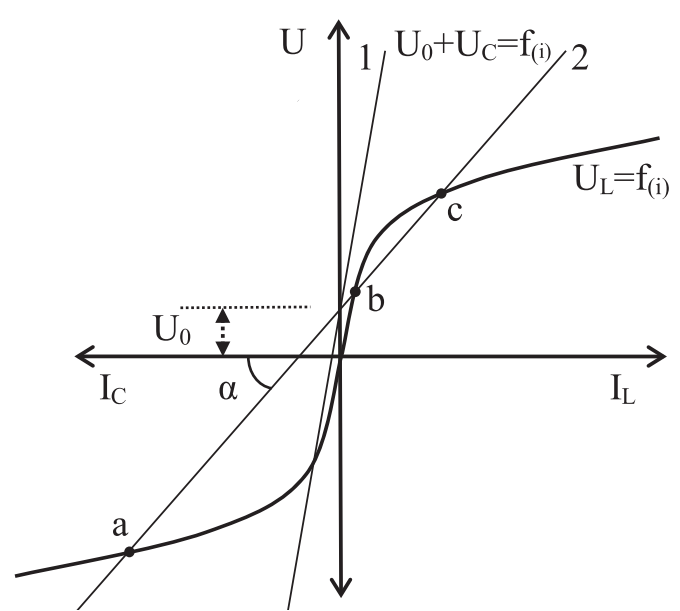

Fig. 6. Graphical solution of (4). Source: [11]

\section{III.Ferroresonance In Distribution Systems}

Conditions favorable to ferroresonance are innumerable given the presence of elements capable of storing electrical charges (overhead lines, shielded cables, capacitor banks,) non-linear inductance in a distribution system and the great variety of network operation configurations. The literature section documents the different cases in which the phenomenon occurs in real networks [10], [13]-[17]. In this section there is an analysis of the most common generic cases for a typical rural distribution system in the region that uses MT / BT-delta / star transformer with earthed rigid neutral.

\section{A. One or two -phase fed Transformer}

In Figs. 7 and 8 circuit configurations conducive to ferroresonance [10] are presented. The phenomenon occurs when a transformer in vacuum or with a very little load feeds on a network with one (Fig. 7) or two phases (Fig. 8).

These circumstances may occur after a fault or operation of a cutoff device: performance of one or two fuses, unipolar reclosers operation, conductor cutoff.

Capacitances involved generally come from overhead lines or shielded cables that feed transformers whose primary windings are connected in delta, in isolated neutral star or earthed neutral. For the schemes of Figs. 7 and 8, the ferroresonant circuit is originated by series capacitances to earth of the open phases (between the opening point and the transformer) and the magnetizing inductance of the transformer.

Fig. 7 shows the case of the opening biphasic where $\mathrm{X}_{\mathrm{T}}$ and $\mathrm{X}_{\mathrm{C}}$ are part of trajectories circulated by the same current. When the values of the resulting series reactances (capacitive and inductive) reach similar values, ferroresonance occurs and surges can occur both in the windings of the transformers $\left(\mathrm{X}_{\mathrm{T}}\right)$ and the supply lines to earth $\left(\mathrm{X}_{\mathrm{C}}\right)$.

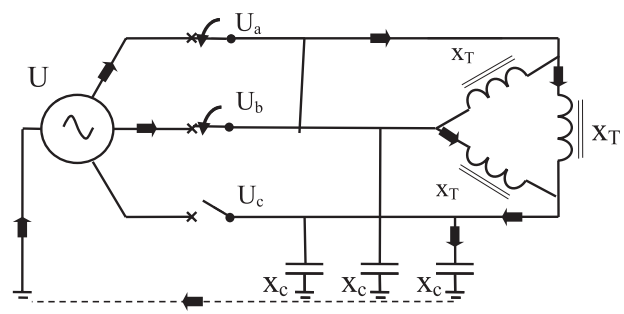

Fig. 7 Single line feeding circuit at risk of ferroresonance.

$$
\text { source: [10] }
$$

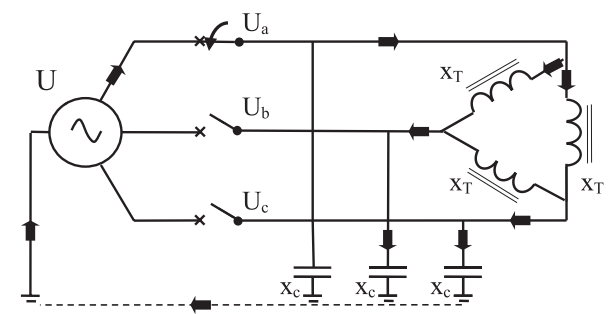

Fig. 8. Two line- feeding circuit at risk of ferroresonance.

Source: [10]

Due to the symmetry of the electrical parameters of the circuit, and not being necessary to consider the capacity to earth of the fed line, the problem can be simplified to the system shown in Fig. 9 [18].

Voltages to earth of phases " $b$ " and " $c$ " take the value shown in (5):

$$
\bar{U}_{b}=\bar{U}_{c}=\frac{\bar{U}_{a}}{j \frac{X_{T}}{2}-j \frac{X_{C}}{2}} \cdot\left(-j \frac{X_{C}}{2}\right)
$$

Mathematically operating on (6):

$$
\bar{U}_{b}=\bar{U}_{c}=\left(\frac{-\frac{X_{C}}{X_{T}}}{1-\frac{X_{C}}{X_{T}}}\right) \cdot \bar{U}_{a}
$$

When observing (6) the $\mathrm{X}_{\mathrm{C}} / \mathrm{X}_{\mathrm{T}}$ ratio approaching 1 , the value of $U_{b}=U_{c}$ tends to infinity, involving high overvoltage values in phases without feeding.

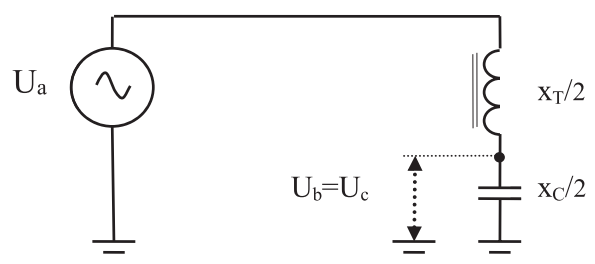

Fig. 9. Circuit with a single feeding line. Simplified. Source: Authors. 


\section{CASE REPORT}

The concession area of the electric company for the distribution of electric power, on which the case is reported, is mostly comprised of rural areas. Farm houses, ranches, and irrigation systems, are the typical loads in low voltage.

In Fig. 10 the scheme of the three-phase trunk distributor which is $4.71 \mathrm{~km}$ of length is shown directly feeding three $100 \mathrm{kVA}$ transformers and from which each monofilament line departs with earth return (LMRT) of the phases $b$ and $c$ with a total of $3 \mathrm{~km}$, similar to the system that have reported faults.

The primary distribution system in $13,2 \mathrm{kV}$ is carried out from a transformer station $33 / 13,2 \mathrm{kV}$ through three wire trunk lines without neutral transport, which can reach $50 \mathrm{~km}$. The typical used conductors are aluminum alloy 25 to $95 \mathrm{~mm}^{2}$ and they are aerially transported in horizontally coplanar arrangement. The loads are single phase, unipolar or bipolar and three-phase transformers in delta-star connection, with its powers being from $5 \mathrm{kVA}$ (single) to 160 kVA (three-phase), with secondary voltage of $380 /$ $220 \mathrm{~V}$. It is also common to find lines LMRT type that, after some kilometers, feed a $5 \mathrm{kVA}$ transformer with simple secondary voltage similar to the previous.

Protective and switching equipment generally used in medium voltage lines are unipolar switches-acting fuses, reclosers, surge arresters, etc.

Fault reports of the represented circuit indicate overvoltage with damage to low voltage loads fed by 5 kVA transformers (T4 and T5) belonging to LMRT lines (Fig. 10). The user of the transformer T5 also noted "strong vibrations" coming from this device.

At the same time, technical staff of the electrical company detects the action of the phase $b$ and $c$ fuses (F) of the $13.2 \mathrm{kV}$ trunk line.

Under these circumstances the company decided to conduct a study of the distribution network involved, in order to find possible causes of technical failure.

\section{Circuit Modeling in Atpdraw}

Simulation and study of transients and ferroresonance phenomena that occurred in electric power systems requires specialized software. The Electromagnetic Transients Program offers in its graphic environment
(ATPDraw), an appropriate medium for the analysis of those phenomena.

The studied circuit lines were modeled from its constituent materials and geometric data with the Line Constants routine (LCC). Being ferroresonance a transient case of low frequency [19] between $0.1 \mathrm{~Hz}$ to $1 \mathrm{kHz}$ [20], according to [20], [21], a multi-phase model of concentrated and constant parameters (PI), including asymmetry of conductors, it is sufficient to model a medium voltage overhead line of a short and less than $50 \mathrm{~km}$ length.

The three phase trunk line is the overhead type with wires of $\mathrm{Al} / \mathrm{Al} 25 \mathrm{~mm}^{2}$ mounted on crossarms IRAM 110 Standard [22] in a horizontal coplanar arrangement at a pole of $7.5 \mathrm{~m}$. height. The monofilament line with earth return (LMRT) is constituted by the same conductor type and is mounted at the same height.

The three-phase transformers are of the three column type and were represented by the Hybrid Model (XFMR), which turn out to be appropriate to represent its transient behavior in low and medium frequency like ferroresonance [12], [23] - [27].

Available data from the three- phase transformers are given in Tables I and II and comply with the provisions of IRAM 2250 standard [28].

Table I. Nominal and Testing Data of A Short Circuit of Three- Phase Transformers

\begin{tabular}{|c|c|}
\hline Power & $100 \mathrm{kVA}$ \\
\hline Voltage & $13.2 / 0.4 \mathrm{kV}$ \\
\hline Connection & Dy11 \\
\hline SC. Voltage & $4 \%$ \\
\hline SC. Losses & $1.75 \mathrm{~kW}$ \\
\hline
\end{tabular}

Source: Usual information featured in a transformer front plate.

Table II. Vacuum Testing Data of Three-Phase Transformers

\begin{tabular}{|c|c|c|}
\hline Testing Voltage \% Un & I Vacuum \% & Vacuum losses $[\mathrm{kW}]$ \\
\hline $100 \%$ & $2,5 \%$ & 0,35 \\
\hline $105 \%$ & $3,5 \%$ & 0,386 \\
\hline
\end{tabular}

Source: Usual information featured in a transformer front plate.

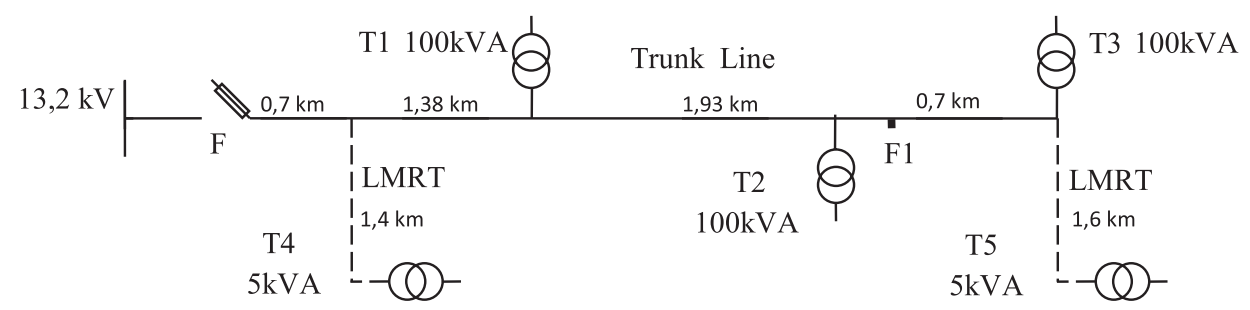

Fig.10. Single line diagram of the radial rural distributor being studied. Source: Authors. 
The single-phase transformers were represented by the Saturable model with the aid of Satura subroutine. Details thereof are given in Table III.

Table III. Nominal and Testing Data of Single Phase Transformers

\begin{tabular}{|l|c|}
\hline Power & $5 \mathrm{kVA}$ \\
\hline Voltage & $7,62 / 0,231 \mathrm{kV}$ \\
\hline SC Voltage & $4,1 \%$ \\
\hline SC Losses & $0,110 \mathrm{~kW}$ \\
\hline I Vacuum & $10,7 \%$ \\
\hline Vacuum Losses & $0,063 \mathrm{~kW}$ \\
\hline
\end{tabular}

Source: Usual information featured in a transformer front plate.

Fig .11 shows the modeled circuit with ATPDraw.

\section{CASE ANalysis}

\section{A. Opening of two Fuses}

The opening simulation of phases $b$ and $c$ fuses was made (according to the facts described by technical personnel of the electrical company) assuming that the transformers are unloaded. The results are shown in the following figures.

Fig. 12 shows the voltages to earth reached by conductors $a, b$ and $c$ of the trunk distributor after the opening of the fuses. Phase $a$ remains with the same values as before the opening, while phases $b$ and $c$ reach maximum pick values up to $20.1 \mathrm{kV}$ in transient (1.93 p.u, in respect to the peak phase voltage of the system) and $16.2 \mathrm{kV}(1.5 \mathrm{pu})$ at steady state in phase $b$.

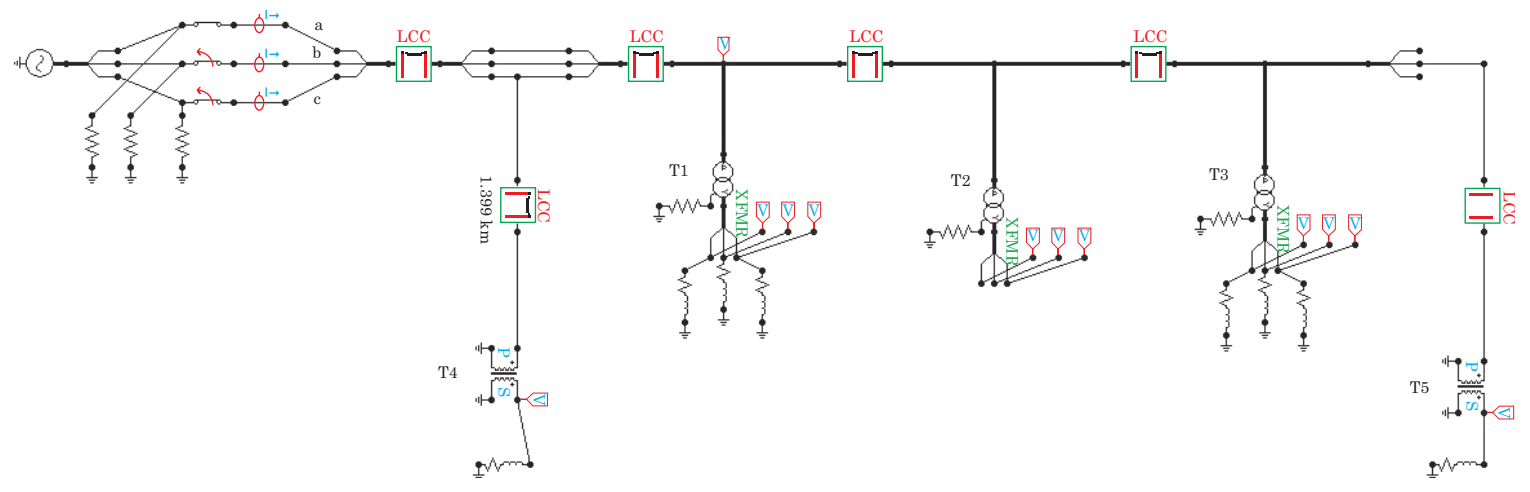

Fig. 11. Scheme of the modeled distributor in ATPDraw .

Source: Authors.

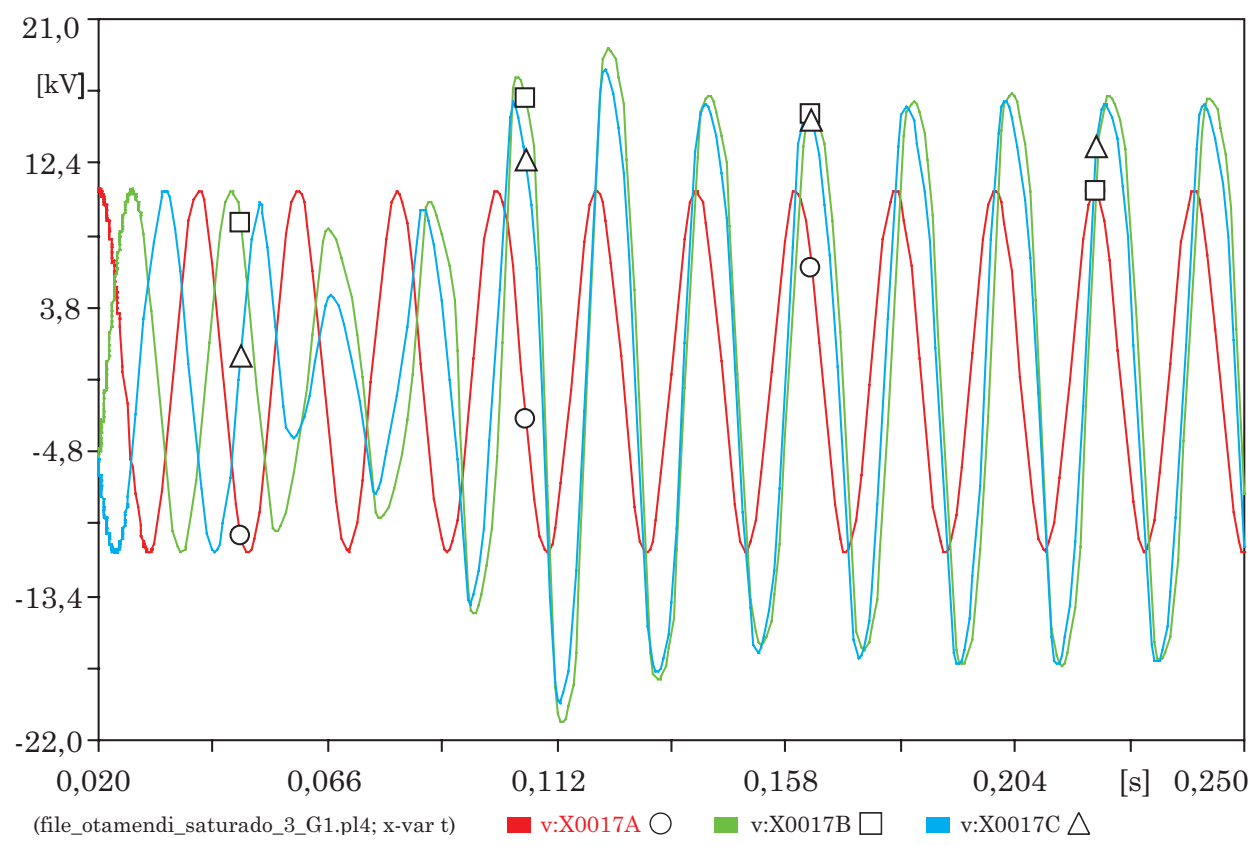

Fig 12. Voltages to earth of $\mathrm{a}, \mathrm{b}$ and $\mathrm{c}$ conductors of the distributor. Opening of two fuses. Source: Authors. 
These tensions, $\mathrm{U}_{\mathrm{b}}$ and $\mathrm{U}_{\mathrm{c}}$, appeared in capacitors $\mathrm{Xc}$ which correspond to the open phases of the circuit of Fig. 7 and they are the ones applied to single-phase transformers (T4, T5.) Fig. 13 shows a resulting simplified circuit diagram.

The voltages developed between phases of the primary windings of the three-phase transformers are shown in Fig. 14. It can be seen that the maximum peak value reached in steady state corresponding to the voltage $a-b$ and is $17 \mathrm{kV}$, which represents $0.91 \mathrm{p} . \mathrm{u}$ expressed in value relative to the peak of the composed voltage of the system. This situation implies an unsaturation regime of the transformer cores.

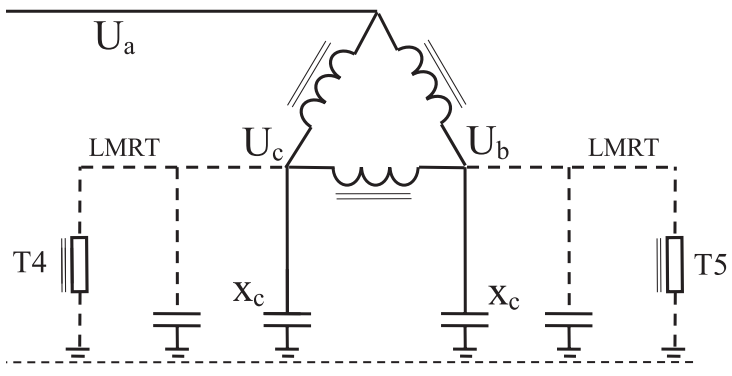

Fig.13. Simplified circuit with opening of phase $a$ and $b$ fuses. Source: Authors

The asymmetry of the circuit, due to the length of power conductors and single-phase transformers, causes both the phase to earth voltages not to be equal and the $b$ - $c$ windings voltage of the three-phase transformers not to be zero, as shown respectively in Figs. 12 and 14.
Fig. 15 shows the voltage waveforms of the low voltage side of the single-phase transformers T4 and T5 fed on phases $c$ and $b$ respectively. The maximum peak voltage in transient is reached in T5 with a value of $583 \mathrm{~V}$ (1.9 p.u.) In steady state, a value of $492 \mathrm{~V}$ is reached (1.6pu in respect to the voltage of peak phase low voltage.)

The simulation results are consistent with the phenomena described by the system users, referring to damage in low voltage installations and "intense vibrations" of single-phase transformers and the absence of these symptoms in the three-phase ones.

According to [15], the ferroresonance phenomenon is manifested in greater magnitude when the load applied to the secondary of the transformer is low value.

For the case of zero load, the behavior is analyzed in the preceding paragraphs.

One possible low load scenario for the distribution network under study may correspond to the rainy season, as the main loads of three-phase transformers are irrigation pumps. In these circumstances and considering some type of residential consumption in farm houses, it may be a charge of $4 \%$ for a single phase (T4) and $1 \%$ for a threephase transformer (T1).

Once the simulation was performed in the set context, it is observed the appearance of an overvoltage of $1.45 \mathrm{p} . \mathrm{u}$ in the peak voltage to earth phase $b$ (Fig. 16) which is reflected in the secondary winding of the single-phase transformer powered by the same phase with a value of $1.5 \mathrm{p} . \mathrm{u}$ (Fig. 17).

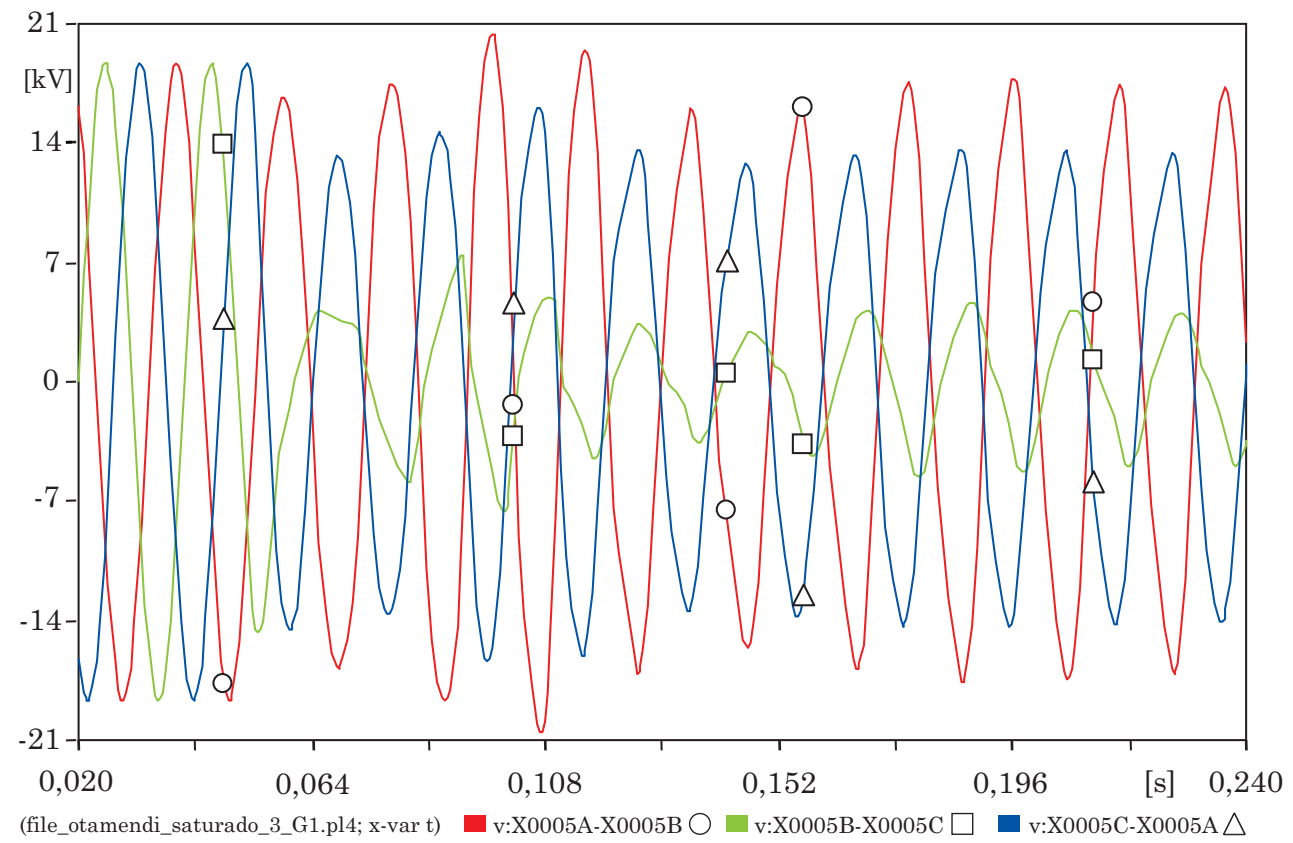

Fig.14. Voltages at the primary windings of three-phase transformers. Opening of two fuses. Source: Authors 


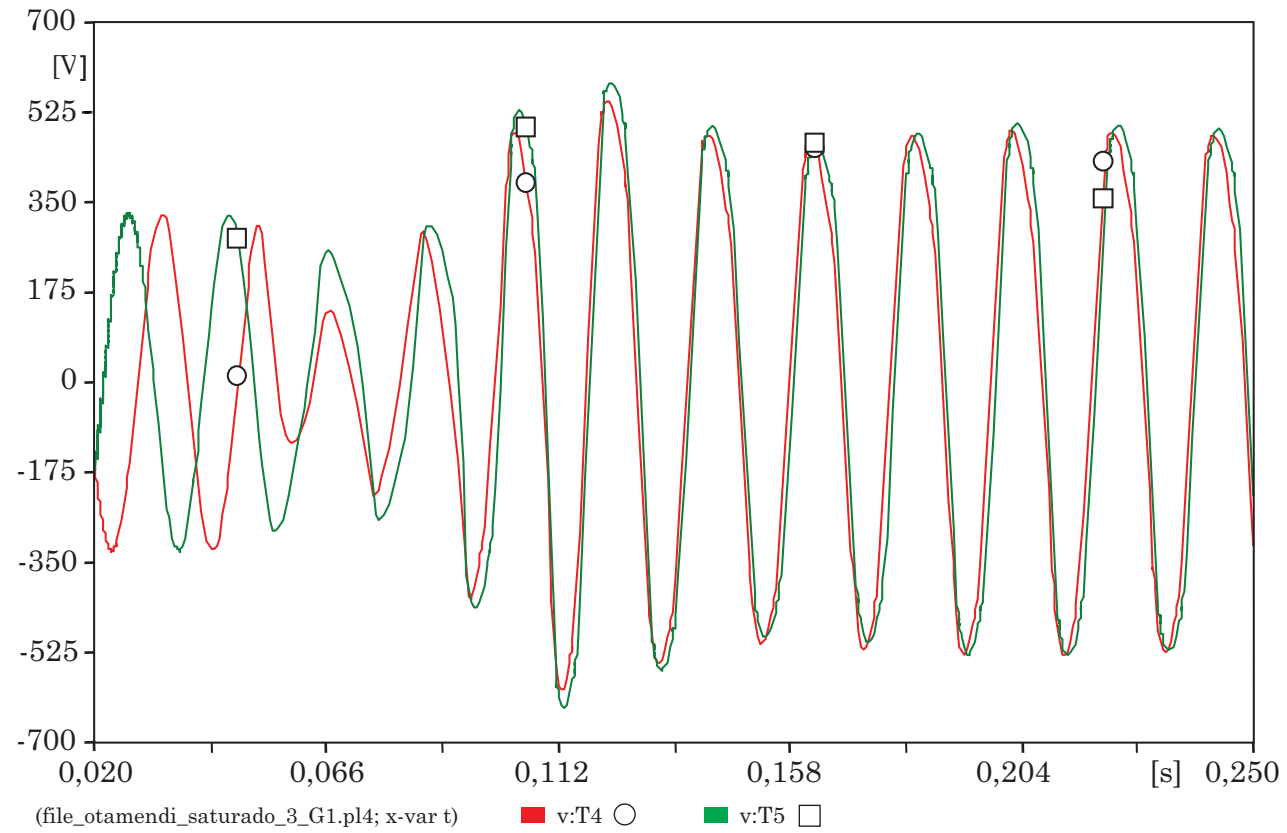

Fig15. Voltage from the low voltage side of single-phase transformers. Opening of two fuses. Source: Authors

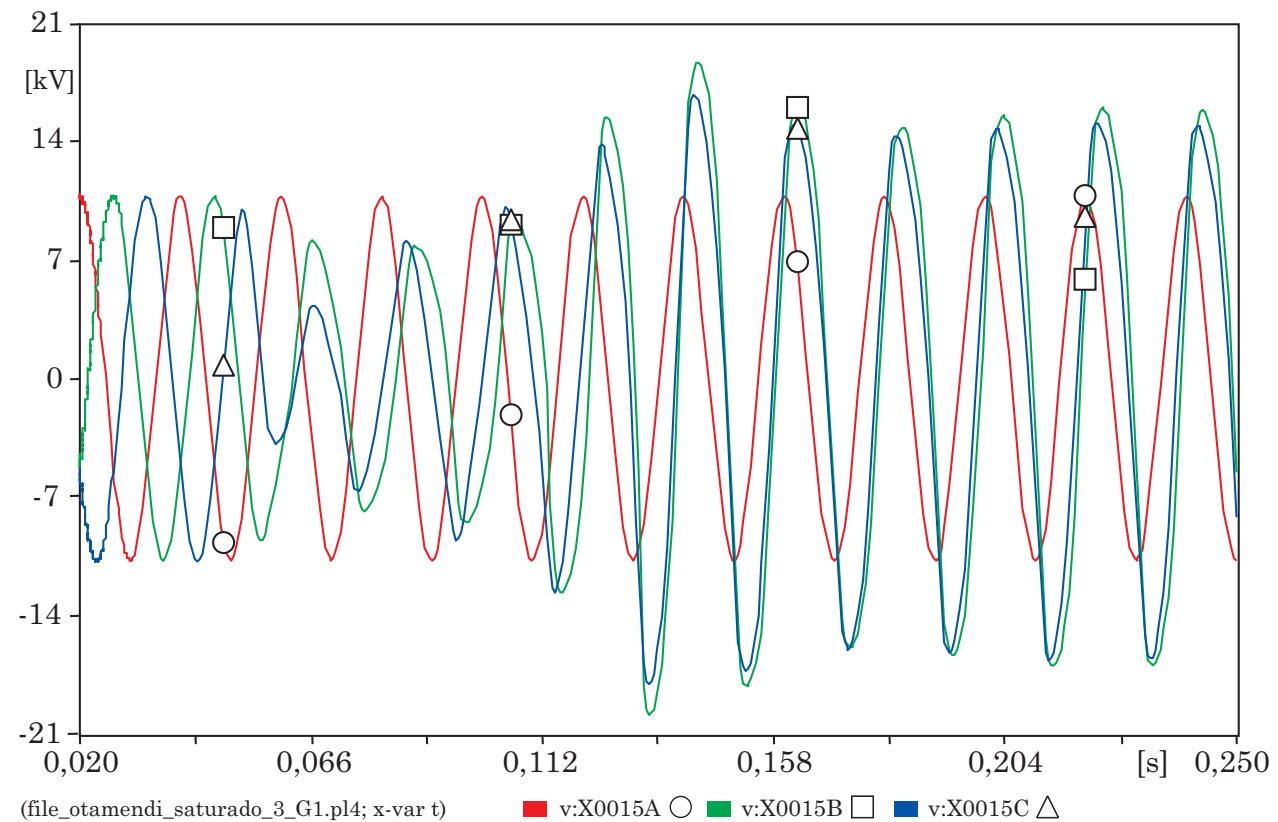

Fig 16. Voltage to earth of conductors $a, b$ and $c$ of the distributor with load $4 \%$ in $\mathrm{T} 4$ and $1 \%$ in $\mathrm{T} 1$. Opening of two fuses.

Source: Authors.

There are multiple variants of loads that can be simulated; in particular, those in which the surge phenomenon is alleviated almost entirely for this cir- cuit; for example, for a load of $6 \%$ for a three-phase transformer (T1). Fig. 18 shows the phase voltages of MT side with a maximum value of 1.06 p.u in $b$. 


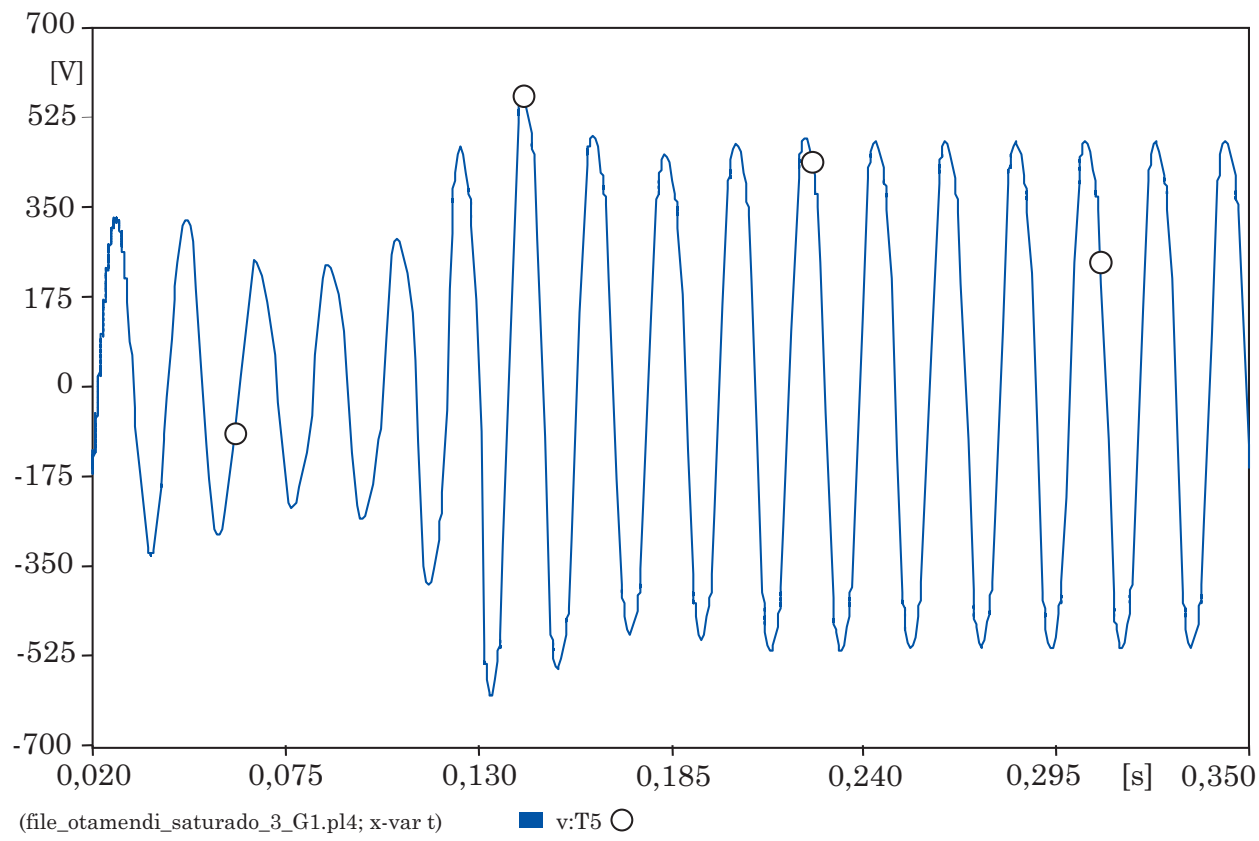

Fig. 17. Voltage in low voltage side of the transformer T5 with load $4 \%$ in $\mathrm{T} 4$ and $1 \%$ in $\mathrm{T} 1$. Opening of two fuses.

Source: Authors

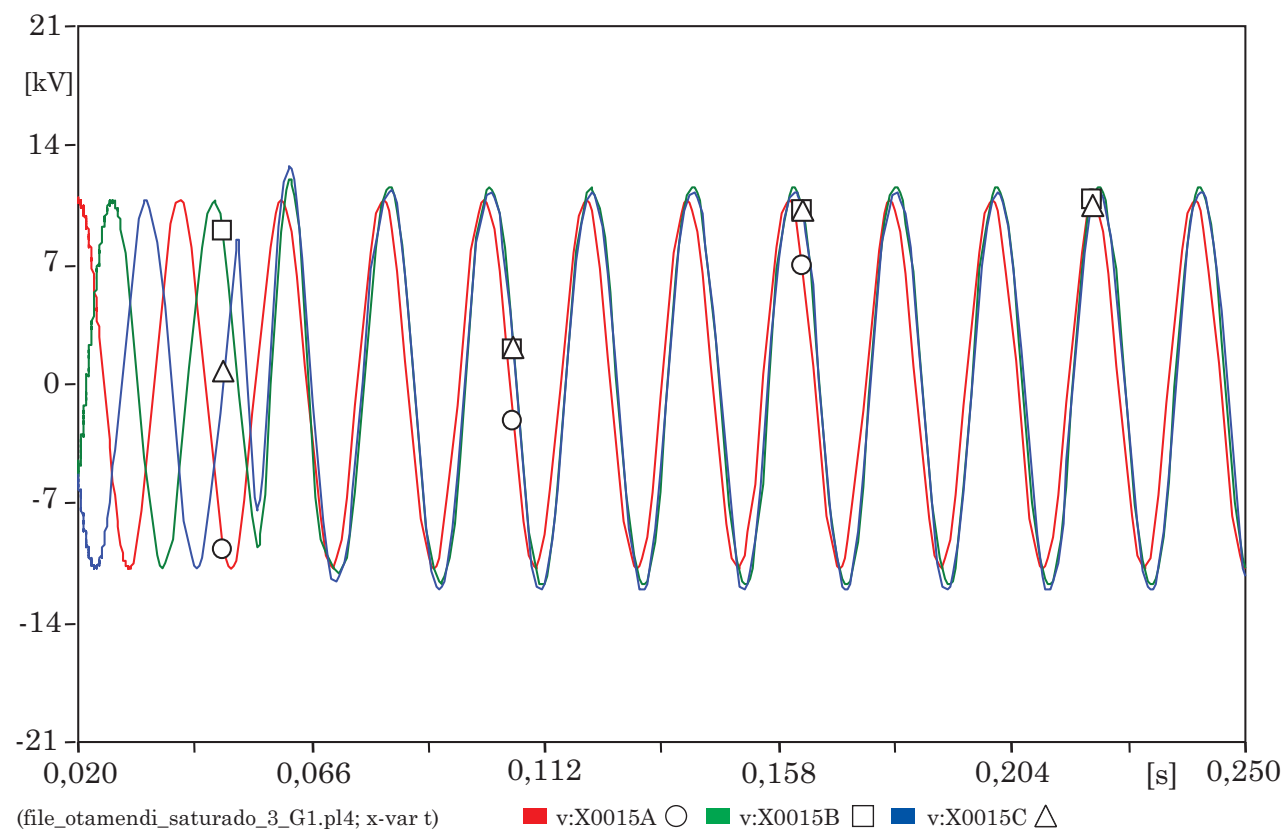

Fig. 18. Voltage to earth of the conductors $a, b$ and $c$ of the distributor with load $6 \%$ in T1. Opening of two fuses.

Source: Authors.

\section{B. Opening a fuse}

For the same load conditions as in the previous sec tion, the opening of a fuse on the $c$ phase was simulated. In none of the presented cases for this circuit configuration, with unloaded transformers (Figs. 


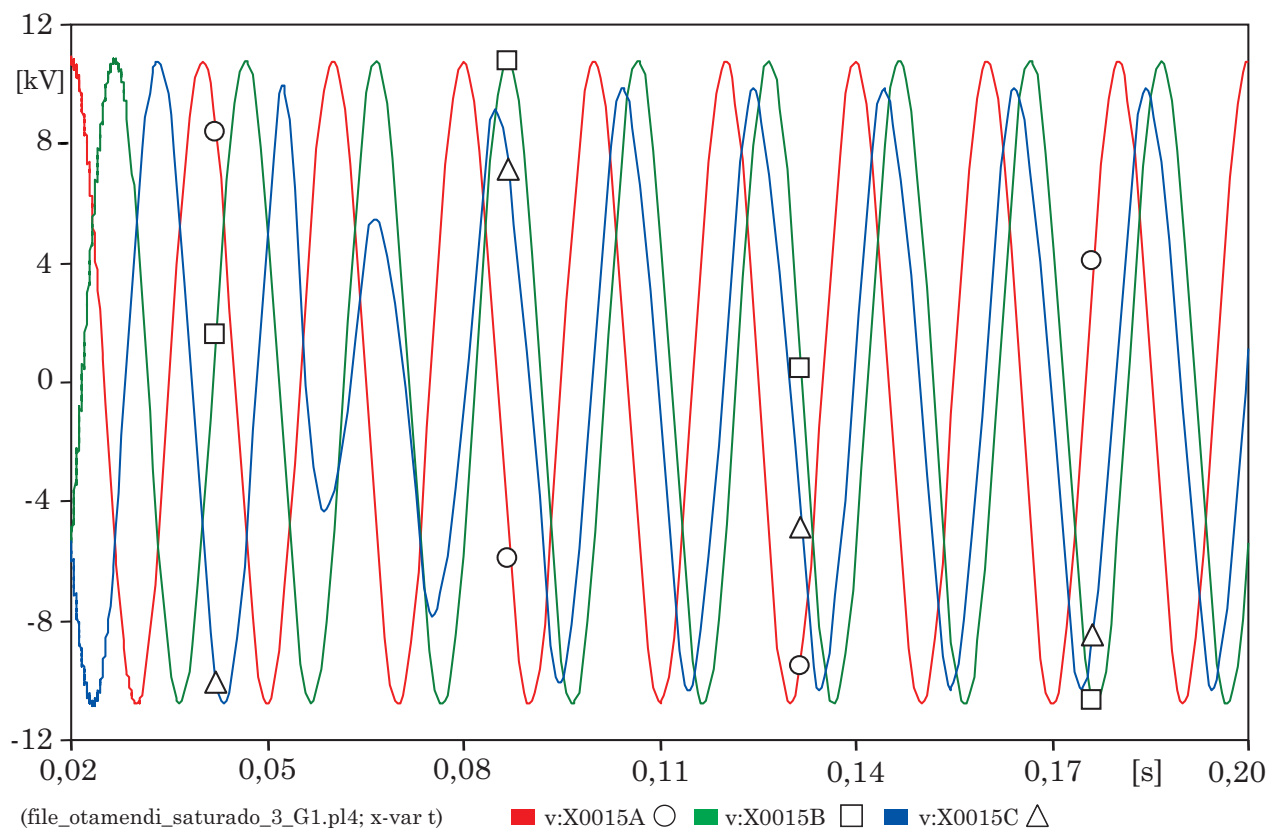

Fig. 19. Voltages to earth of the $a, b$ and $c$ conductors of the distributor. No load; opening of a fuse. Source: Authors.

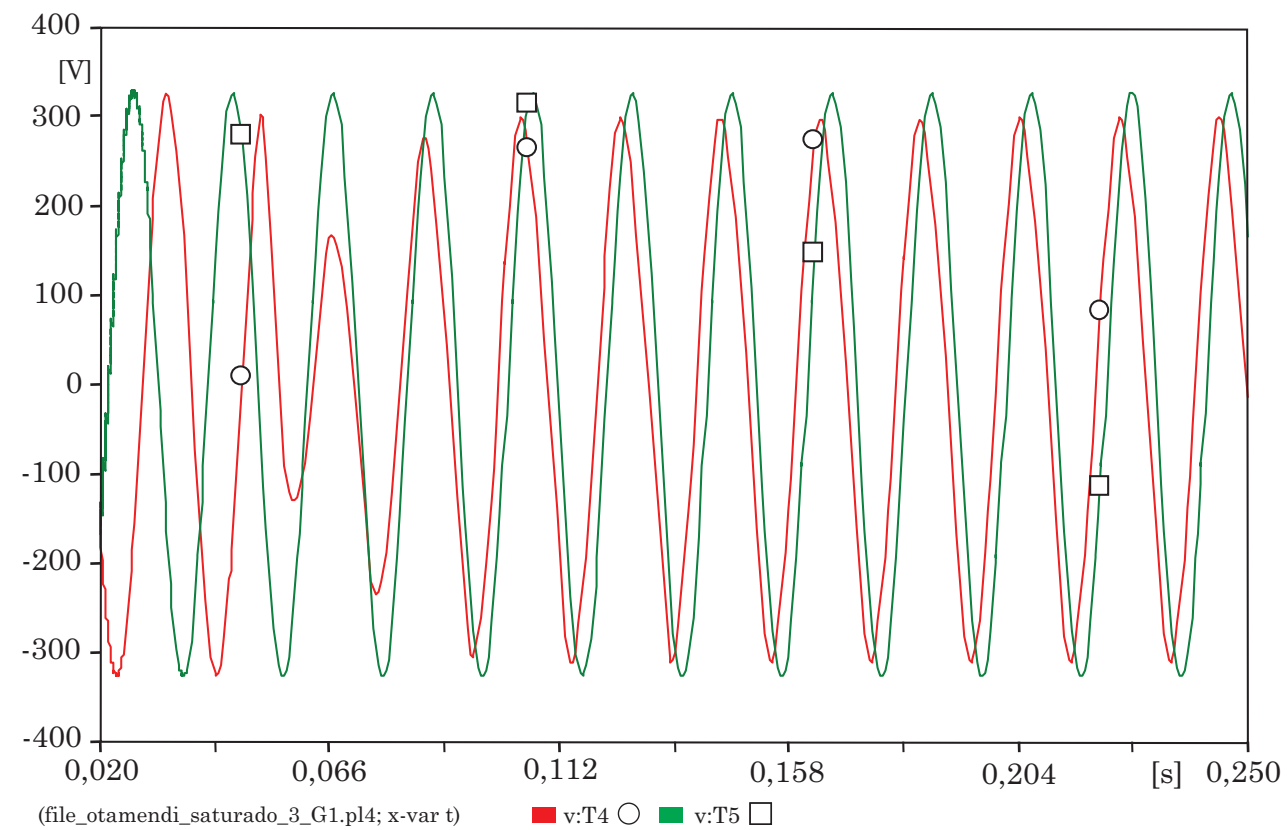

Fig. 20. Voltage of the low voltage side of single-phase transformers. No load; opening of a fuse. Source: Authors.

19 and 20) and with a load of $4 \%$ in T4 and $1 \%$ in T1 (Figs. 21, 22), overvoltage effects are observed. By contrast, the voltage in the open phase, both in the medium and low voltage side of transformer T4, presents lower values than the nominal steady-state values. 


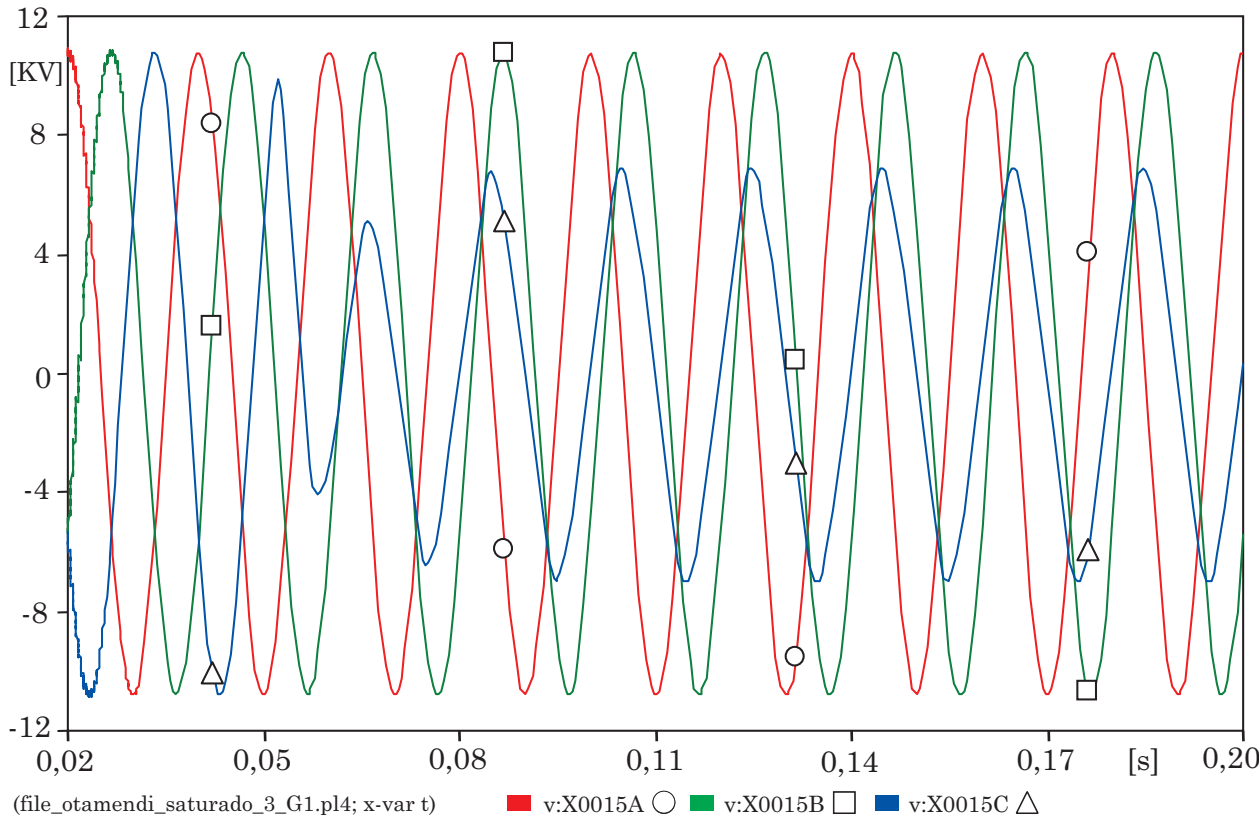

Fig. 21. Voltages to earth of conductors $a, b$ and $c$ of the distributor with load $4 \%$ in $\mathrm{T} 4$ and $1 \%$ in $\mathrm{T} 1$. Opening of a fuse. Source: Authors.

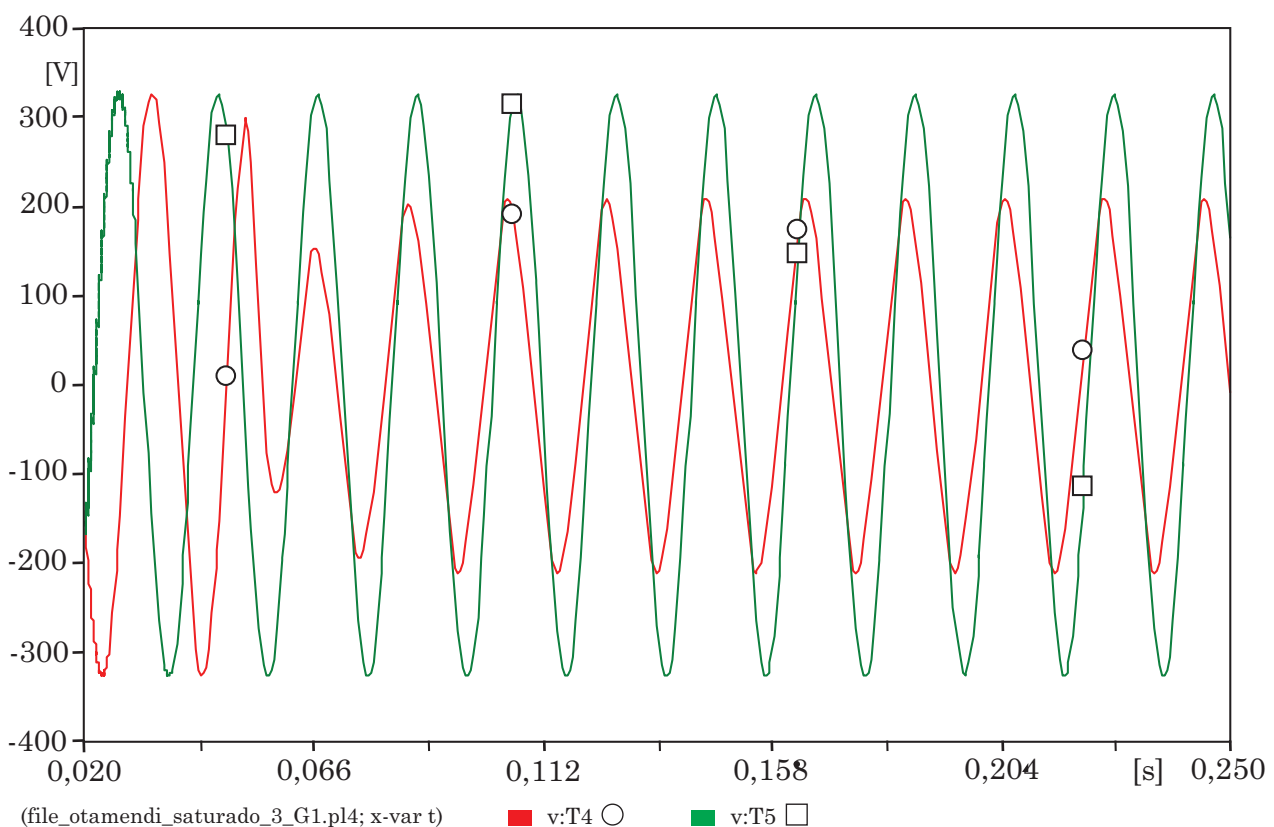

Fig. 22. Voltages in low voltage side of single phase transformers with load $4 \%$ in $\mathrm{T} 4$ and $1 \%$ in $\mathrm{T} 1$. Opening of a fuse. Source: Authors

VII. Mitigation Guidelines

Before thinking about mitigation measures of the ferroresonance phenomenon, it is convenient to fo- recast these situations from the design of the distribution network. This entails, inter alia, parameters and length of lines; features and connection of the transformers; type and location of switching ele- 
ments; and load conditions of users. The selection of the appropriate characteristics and network topology can help identify risk configurations.

Considering the cases above discussed, and depending on the parameters of the intervening components of the electrical network, a radical solution is the use of three-pole switching devices. Unfortunately this solution is not always technically and /or economically convenient.

A relevant parameter turns out to be the length of feeder lines downstream of a switching position (and / or protection) with unipolar opening. On such length depends that the involved capacitance to earth takes on values that can lead to the circuit resonance paired with the transformers being fed The number and location of switching devices on trunk and branch lines deserves to be evaluated.

It is suitable to have an adequate coordination between the protection devices of transformers and those located upstream in the supply line. In case of own faults or in the secondary of any transformer, opening one or two protection fuses, will not create any situation susceptible to ferroresonance; whereas, it can occur if the opening is done in the upper protection as already discussed.

If in the trunk line of the discussed network, switching elements with automatic cutoff are positioned downstream of the start of the distributor and coordinated with fuses $\mathrm{F}$, it's possible, in the event of a fault posterior to those, and if any of its elements is opened, to achieve a change in the capacitance / inductance ratio at stake in a probable series resonant circuit.

Once analyzed the different locations where the above conditions prove effective, it was found that a possible place to locate a fuse in the trunk line, is point $\mathrm{F} 1$ at $0.2 \mathrm{~km}$ downstream of the transformer T2 (Fig. 10.) It is observed that the surge phenomenon hardly occurs when the same type of fault that occurred downstream of F1 with opening phases $b$ and $c$ was simulated (Fig. 23.)

$\mathrm{T} 1$, T2, and T3 transformers, as mentioned in section IV, are used to power irrigation pumps in dry seasons. In this sense the circuit behavior was analyzed in the event that any of the transformers would be left out of service. The failure was simulated again with the transformer $\mathrm{T} 1$ disconnected. The results are shown in Fig. 24 wherein as seen there is virtual absence of surges in the circuit. By performing the same tests with the T2 and T3 transformers, the same results are obtained.

On the other hand, other mitigation actions are feasible to consider. Having unipolar cutoff elements in the network, the occurrence of ferroresonance conditions can be avoided with proper sequence of maneuvers at the time of operation, particularly when transformers are operating in vacuum or lightly loaded.

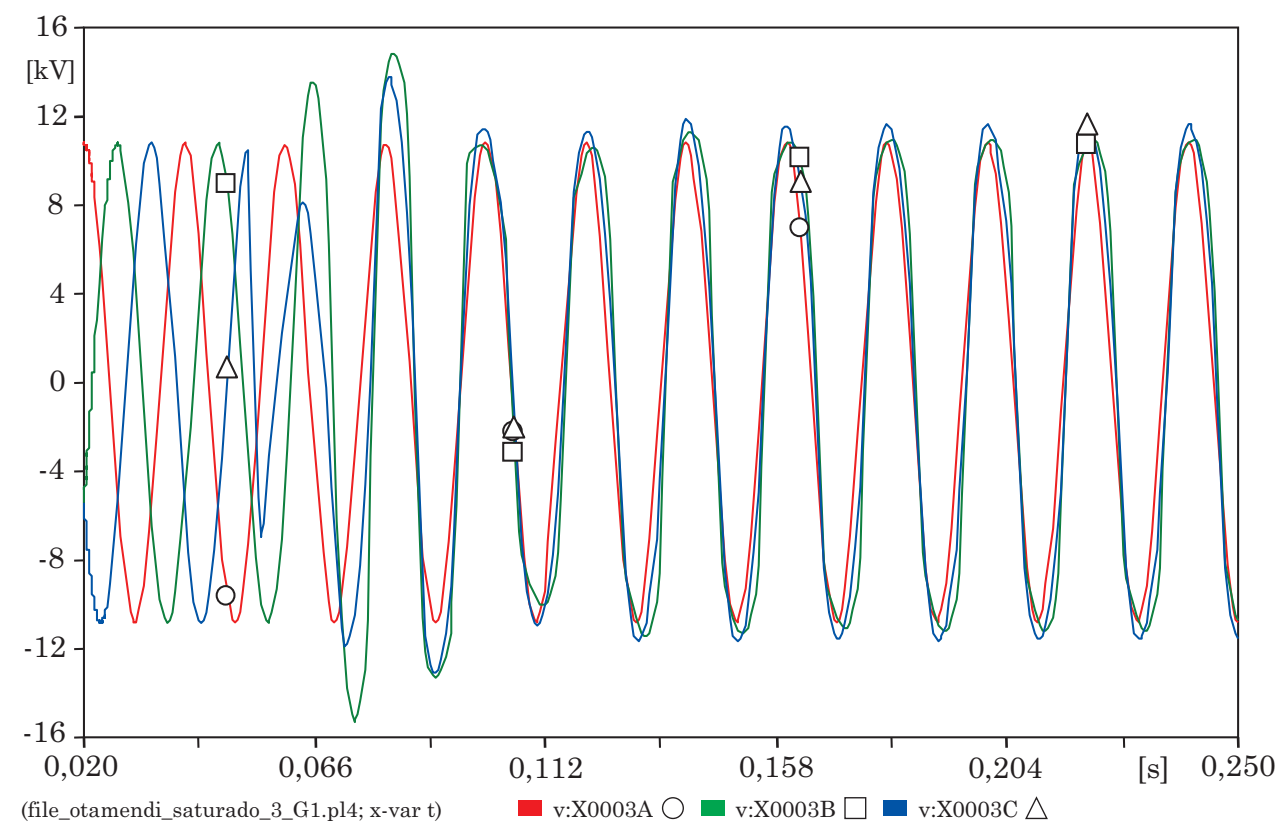

Fig. 23. Earthed voltages of conductors a, b and c of the distributor in point F1 with opening of two fuses in the same place. No load. Source: Authors. 


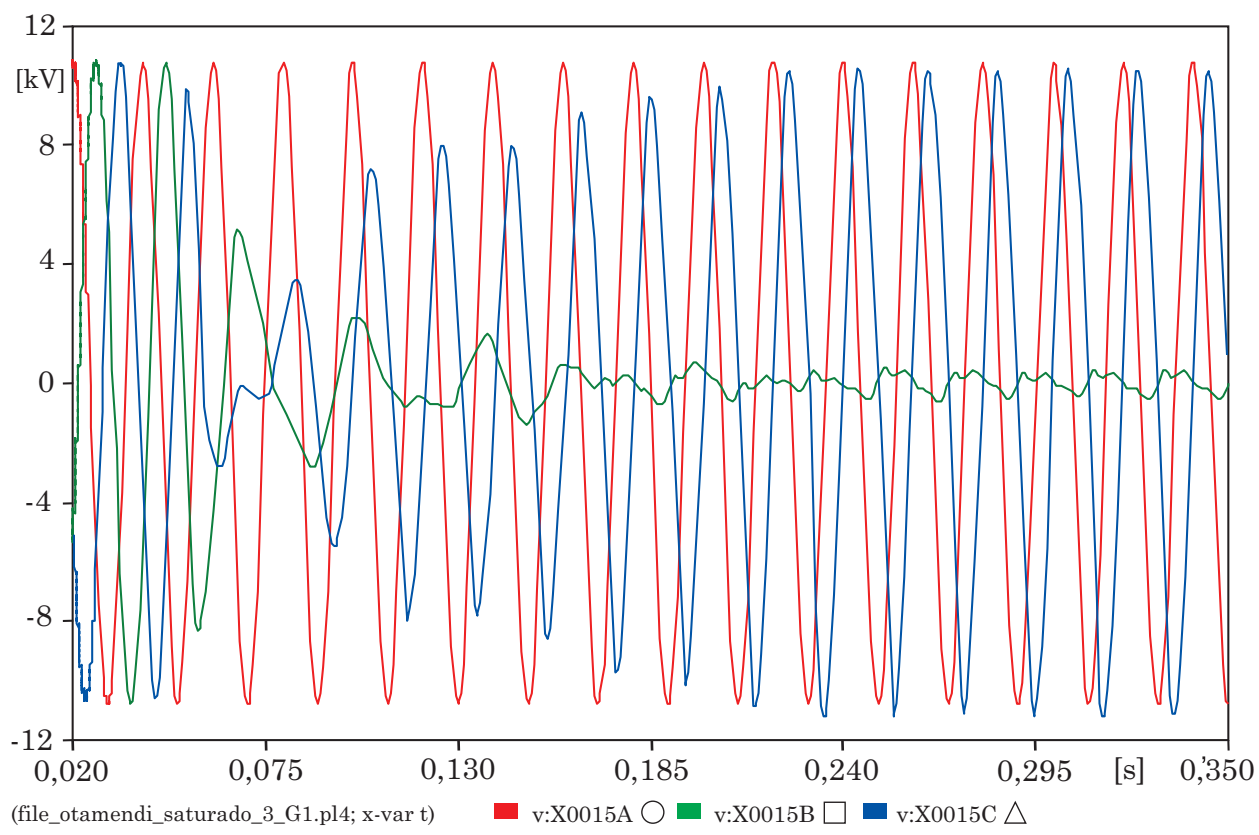

Fig. 24. Earthed voltages of conductors a, b and c of the distributor. Disconnection of transformer T1. Source: Authors.

Fig. 25 shows a transformer with elements of local protection (T) fed from a line with its fuse switch disconnectors positioned upstream (S). It is about using a proper sequence and thus prevent the earthed capacitances of the feeder with the inductance of the primary winding of the transformer from being in series. For the energizing of the circuit, it is needed in first instance, the closure of the switch disconnectors $\mathrm{S}$ of the line, and then the transformer protection $\mathrm{T}$. The reverse sequence is suitable for de-energizing.

In reference [29] it is verified the existence of a correlation between core loss of transformers and capacitance needed to maintain a condition of ferroresonance. From the same reference it can also be noted that transformers with higher losses in the core are candidates to be located in those places where the network conditions are conducive to the emergence of the phenomenon.

As it was discussed above, when transformers have some load on the secondary, the effects of ferroresonance are attenuated considerably. Therefore, to make a maneuver with unipolar cutoff elements, it is desirable to do it with loads connected to the transformers and with the least possible delay between the cutoff elements of each phase.

\section{Conclusions}

The study of the actual distribution system consisting of rural overhead lines, allowed verifying that under certain operating conditions and depending

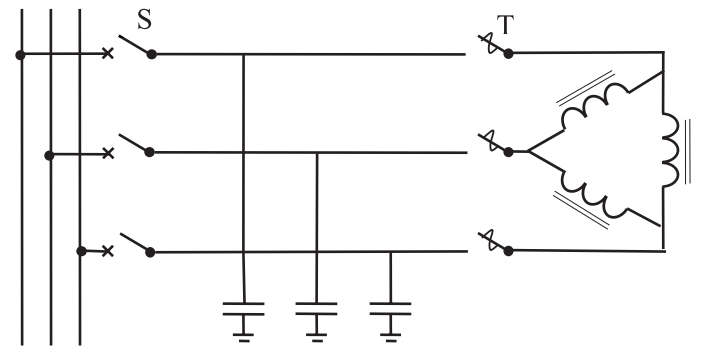

Fig. 25. Scheme for switching energization / de-energization. Source: Authors

on the characteristics of its components, the phenomenon of ferroresonance can be present.

The results of the simulation of different events for switching by ATPDraw program showed that ferroresonance presents overvoltage values that can be harmful to the single-phase transformers with earth return connected to the phases on which the circuit was opened. These effects coincided with those observed by operators and customers of the electricity distributor.

For the particular case of this analyzed circuit, it was found that when simulating the opening of the phase fuses $b$ and $c$ with any of the disconnected three-phase transformers, the overvoltage phenomenon is almost nonexistent. With this fact and as a guideline to mitigate the phenomenon, it is suggested the disconnection of those transformers for irrigation during the rainy season. 
Furthermore, it was verified that locating fuse elements downstream of the start of the distributor and coordinated with the precedent ones, in case of a similar failure beneath them, would not allow ferroresonance to appear, which is another example of mitigation.

\section{REFERENCES}

[1] G. Buigues, I. Zamora, V. Valverde, A. J. Mazón, and J. I. S. Martín, "Ferroresonance in Three-Phase Power Distribution Transformers: Sources, Consequences and Prevention," in 19th International Conference on Electricity Distribution, 2007, pp. 21-24.

[2] M. Roy and C. K. Roy, "A Study on Ferroresonance and Its Depedence on Instant of Switching Angle of the Source Voltage," in International Conference on Power Systems ICPS'09, 2009, pp. 1-6. DOI:10.1109/ icpws.2009.5442704

[3] K. Miličević, I. Rutnik, and I. Lukačević, "Impact of Initial Conditions and Voltage Source on the Initiation of Fundamental Frequency Ferroresonance," in 12th WSEAS International Conference on SYSTEMS, 2008, pp. 22-24.

[4] J. B. Wareing and F. Perrot, "Ferroresonance overvoltages in distribution networks," in IEEE Colloquium: Warning! Ferroresonance Can Damage Your Plant, 1997, pp. 1-5. DOI:10.1049/ic:19971178

[5] S. Santoso, R. C. Dugan, T. E. Grebe, and P. Nedwick., "Modeling Ferroresonance Phenomena in an Underground Distribution System," in International Conference on Power Systems Transients IEEE IPST '01, 2001, pp. 240-245.

[6] L. B. Crann and R. B. Flickinger, "Overvoltages on 14.4/24.9-Kv Rural Distribution Systems," IEEE Trans. Power Appar. Syst., vol. 73, no. 3, pp. 1208-1212, 1954. DOI:10.1109/AIEEPAS.1954.4498949

[7] W. M. Edmunds and L. B. Crann, "Operating Experience With 14.4/24.9 Kv as a Rural Distribution Voltage," III Trans. Am. Inst. Electr. Eng., vol. 75, no. 3, 1956. DOI:10.1109/AIEEPAS.1956.4499265

[8] R. Hopkinson, "Ferroresonance During Single-Phase Switching of 3-Phase Distribution Transformer Banks," IEEE Trans. Power Appar. Syst., vol. 84, no. 4, pp. 289293, 1965. DOI:10.1109/TPAS.1965.4766193

[9] P. Sakarung, T. Bunyagul, and S. Chatratana, "Investigation and Mitigation of Overvoltage Due to Ferroresonance in the Distribution Network," J. Electr. Eng. Technol., vol. 2, no. 3, pp. 300-305, 2007. DOI:10.5370/ JEET.2007.2.3.300

[10] P. Ferracci, La ferrorresonancia, Schneider Electric, 1997, pp. $12-20$

[11] L. A. Siegert, Alta Tensión y Sistemas de Transmisión, Caracas: Limusa, 1989.

[12] J. A. Corea-Araujo, F. Gonzalez-Molina, J. A. MartinezVelasco, J. A. Barrado-Rodrigo, and L. Guasch-Pesquer, "An EMTP-Based Analysis of The Switching Shift Angle Effect During Energization/de-Energization in the Final Ferroresonance State," in International Conference on Power System Transients (IPST), 2013.

[13] D. Jacobson, "Examples of ferroresonance in a high voltage power system," IEEE Power Eng. Soc. Gen. Meet., vol. 2, pp. 1206-1212, 2003. DOI:10.1109/PES.2003.1270499
[14] V. Valverde, G. Buigues, A. J. Mazón, I. Zamora, and I. Albizu, "Ferroresonant Configurations in Power Systems," in International Conference on Renewable Energies and Power Quality (ICREPQ'12), 2012.

[15] E. G. Vinson, A. Jurado, and N. Lemozy., "Ferroresonancia en Transformadores de Distribución. Influencia de sus Características, Secuencia de Maniobra y Carga Secundaria," in VII Congreso Latinoamericano de Generación y Transporte de Energía Eléctrica, 2007, p. 7.

[16] G. Mokryani, M. R. Haghifam, H. Latafat, P. Aliparast, and A. Abdollahy, "Analysis of Ferroresonance in a $20 \mathrm{kV}$ Distribution Network," in 2nd International Conference on Power Electronics and Intelligent Transportation System (PEITS), 2009, pp. 31-35. DOI:10.1109/peits.2009.5407008

[17] W. Chunbao, T. Lijun, and Q. Yinglin, "A study on factors influencing ferroresonance in distribution system," in 4th International Conference on Electric Utility Deregulation and Restructuring and Power Technologies (DRPT), 2011, pp. 583-588. DOI:10.1109/ drpt.2011.5993960

[18] J. Viqueira Landa, Redes eléctricas, 2nd ed. México: Representaciones y Servicios de Ingeniería, 1973.

[19] Study Committee 33, "Guidelines for Representation of Network Elements when Calculating Transients," in International Conference on Large High Voltage Electric Systems, 1988.

[20] J. A. Martinez-Velasco, Power System Transients: Parameter Determination, 1st ed. Estados Unidos: CRC Press, 2009

[21] S. P. Ang, "Ferroresonance Simulation Studies of Transmission Systems," University of Manchester, 2010.

[22] Instituto Argentino de Certificación y Normalización, Norma IRAM 2250. Argentina, 2005.

[23] B. Mork, F. Gonzalez, D. Ishchenko, and D. L. Stuehm, "Hybrid Transformer Model for Transient Simulation-Part I: Development and Parameters," in IEEE Trans. Power Deliv., vol. 22, no. 1, pp. 248-255, 2007. DOI:10.1109/TPWRD.2006.882999

[24] L. B. Viena, F. A. Moreira, N. R. Ferreira, and N. C. de Jesus, "A Comparative Analysis of Transformer Models Available in the ATP Program for the Simulation of Ferroresonance," in International Conference on Power Systems Transients (IPST2011), 2011. DOI:10.1109/ tdc-la.2010.5762966

[25] J. A. Martinez and B. A. Mork, "Transformer Modeling for Low- and Mid-Frequency Transients-A Review," IEEE Trans. Power Deliv., vol. 20, no. 2, pp. 16251632, Apr. 2005. DOI:10.1109/TPWRD.2004.833884

[26] H. K. Høidalen, B. A. Mork, F. Gonzalez, D. Ishchenko, and N. Chiesa, "Implementation and verification of the Hybrid Transformer model in ATPDraw," Electr. Power Syst. Res., vol. 79, no. 3, pp. 454-459, Mar. 2009. DOI:10.1016/j.epsr.2008.09.003

[27] L. M. Lobo, "Modelo de transformadores en saturación utilizando funciones de cálculo de parámetros en EMTP-RV," Ing. Rev. Univ. Costa Rica, vol. 24, no. 2, pp. 105-116, 2014. DOI:10.15517/ring.v24i2.8251

[28] Ente Nacional Regulador de la Electricidad, Resolución ENRE 0444/2006. Argentina, 2006.

[29] R. A. Walling, "Ferroresonance in low-loss distribution transformers," in 2003 IEEE Power Engineering Society General Meeting (IEEE Cat. No.03CH37491), 2003, vol. 2, pp. 1220-1222.DOI:10.1109/PES.2003.1270502 